\title{
Temperature Dependences of IR Spectral Band Frequencies of Soils
}

\author{
Dmitry S. Volkov', Olga B. Rogova ${ }^{1,2}$, Mikhail A. Proskurnin ${ }^{1}$ \\ ${ }^{1}$ Chemistry Department of M.V. Lomonosov Moscow State University \\ Leninskie Gory, 1-3, GSP-1, 119991, Moscow, Russia, \\ dmsvolkov@gmail.com; proskurnin@gmail.com,michael@analyt.chem.msu.ru \\ ${ }^{2}$ Department of Chemistry and Physical Chemistry of Soils, V.V. Dokuchaev Soil Science Institute \\ Pyzhevsky per., 7/2, 119017, Moscow, Russia \\ obrogova@gmail.com
}

\begin{abstract}
Temperature dependences of the IR spectra of different size aggregate fractions of chernozem and soddy podzol soils in the range $25-215^{\circ} \mathrm{C}\left(298-488 \mathrm{~K}\right.$ with a step of $2.5^{\circ} \mathrm{C}$ by heating in the air) for different agriculture use samples were investigated. The band maximum temperature dependence can be much more stable to experimental conditions and thus serve as a new quantitative parameter characterizing soils with IR spectroscopy. For the first time, a reversible change in the frequencies of the band maxima in IR spectra upon heating was observed, which can be interpreted as forming structures with a particular order in the dry state. The difference in the behavior of the lattice vibrations of quartz, hydrogen bonds, and organic matter that can be used to differentiate soils was found and discussed.
\end{abstract}

Keywords: FTIR, infrared spectroscopy, temperature-dependent infrared spectra, chernozem, soddy podzolic

\section{Introduction}

Apart from bulk soil properties, studies on the roles of agrogenesis and anthropogenesis on the changes in physical and physicochemical properties of soils have become increasingly topical [1]. The required problem is assessing soil fractions and structure composition, including changes at meso- and microaggregate levels [2,3]. Solving these problems requires highly informative methods because soils are complex ensembles of inorganic matrices and organic matter [4]. Despite the simplicity and versatility, the information from conventional IR spectroscopy is somewhat limited and constrained by qualitative comparison of the functional-group composition. However, IR spectroscopy potential for soils is far from being exhausted. Changes in the vibrational spectra of solid samples upon heating or cooling provide information on polymorphic transformations, structural phase transitions, intramolecular interactions, and second-order phase transitions. Also, temperature-dependent IR spectra may reveal changes in the band intensities, positions, shape, and width [5]. This work aims to demonstrate the capabilities of temperature-dependence IR spectroscopy to increase the information level. The IR spectra of chernozem and soddy podzol soils were studied upon continuous heating from 25 to $215^{\circ} \mathrm{C}$. All the experiments (temperature dependence, centrifugation, and matrix assignment) were used previously but never combined.

\section{Materials and Methods}

Samples of the sod-podzolic soil were taken on the experimental field of the Zelenograd station of the V.V. Dokuchaev Soil Institute (village of Eldigino, Moscow region, Russia). The soil is agrosod-podzolic medium loamy (sod-podzolic, Umbric Albeluvisols Abruptic by WRB 2006) formed on a mantle loam, underlain at a depth of 2-3 m with non-carbonate moraine. Since 2011, the field has not been plowed; a fallow has formed (Sonchus arvensis, Festuca pratensis, Phleum pratense, Dactylis glomerata). Samples were taken in 2016, the coordinates of the sampling site are $56^{\circ} 07^{\prime} 56^{\prime \prime} \mathrm{N} 37^{\circ} 48^{\prime}$ 09" E. Sludge content in the upper (arable) horizon of unflushed full-profile sod-podzolics varies from 10 to $16 \%$ (Sorokina et al., 2013). Minerals dominate the clay fraction mineralogical composition with a rigid structure (hydromica, kaolinites, chlorites in the upper part). The total mineral content varies with the profile as 15-18\% (Travnikova et al., 2010); organic carbon content, $1.37 \% \mathrm{w} / \mathrm{w} ; \mathrm{pH} 5.96$. Typical chernozems (heavy loamy) of the Kursk region (Russia) with a significantly different history and intensity of agricultural use: native (intact, annually mown) steppe vegetation, permanent bare fallow since 1964, shelterbelt since 1964, arable cropland under wheat, cultivated without crop rotation since 1964 [6]. Samples 
were taken on the territory of the long-term field experiment of the Kursk Research Institute of Agricultural Production and V.V. Alekhin Tsentralno-Chernozemny Nature Reserve of Russia. The granulometric composition of the soil is heavy silty-clay loam. Main components: quartz, 35-40\%; illites, $12-15 \%$; smectites, $12-15 \%$; and total organic carbon, $4-6 \%$. The humus horizon $(\mathrm{A}+\mathrm{AB} 1)$ is $105-130 \mathrm{~cm}$. Soil boiling after the addition of $10 \% \mathrm{HCl}$ begins at a depth of $65-70 \mathrm{~cm}$. The arable layer bulk density $(0-30 \mathrm{~cm})$ ranges from 1.20 to $1.25 \mathrm{~g} / \mathrm{cm}^{3}$ [7]. General soil samples with a mass of $2 \mathrm{~kg}$ were taken at the end of May 2017 from sections $1.5 \mathrm{~m}$ deep along genetic horizons and, in addition, with a step of $10 \mathrm{~cm}$. For this study, the topsoil layer, $0-10 \mathrm{~cm}$, was taken. General samples were dried for two weeks in air, then stored at room temperature. An average $0.5 \mathrm{-kg}$ sample was taken from the corresponding total sample, separated into aggregate fractions by dry sieving. The annually mown native steppe site is covered with natural steppe vegetation on V.V. Alekhin Tsentralno-Chernozemny Nature Reserve of Russia; it is a sample of intact typical chernozem. Samples were taken from the section with coordinates $51^{\circ} 34^{\prime} 13.6^{\prime \prime} \mathrm{N} 36^{\circ} 05^{\prime} 23.1^{\prime \prime} \mathrm{E}$.

IR spectra were recorded on a Bruker Vertex 70 single-beam IR Fourier spectrometer (Germany). The spectrometer and accessories were continuously purged with $-70^{\circ} \mathrm{C}$ dew point air (produced by a PG28L Purge Gas Generator, PEAK Scientific) with a flow of $500 \mathrm{~L} / \mathrm{h}$. The overall laboratory temperature was maintained at $23{ }^{\circ} \mathrm{C}$ with an allowable variation of $\pm 1{ }^{\circ} \mathrm{C}$ using an air conditioner. A GladiATR ${ }^{\mathrm{TM}}$ single reflection attenuated total internal reflection accessory with a diamond crystal (Pike Technologies, USA) was used for spectra registration. A background signal was recorded before each new sample. The spectra were recorded using a wide-range silicon beam splitter in the range of 4000-100 $\mathrm{cm}^{-1}$; the baseline was not corrected. Source MIR, globar; beamsplitter, $\mathrm{KBr}$; aperture, $8 \mathrm{~mm}$; detector, room temperature DLaTGS; scanner velocity, $10 \mathrm{kHz}$; resolution, $2 \mathrm{~cm}^{-1}$; sample and background scans, 128, spectral range, 4000-400 $\mathrm{cm}^{-1}$, acquisition mode, double-sided, forward-backward. Before recording the spectra with heating, an empty ATR crystal spectrum was recorded at $25^{\circ} \mathrm{C}$ as a background. Then, a small amount of the sample was placed on its surface, pressed with a screw, and controlled heating was started at a rate of $0.25{ }^{\circ} \mathrm{C} / \mathrm{min}$ from $25^{\circ} \mathrm{C}$ to $215^{\circ} \mathrm{C}$; spectra registration step is $2.5^{\circ} \mathrm{C}$. After heating to $215^{\circ} \mathrm{C}$, the sample was cooled to $25^{\circ} \mathrm{C}$ in the same manner at a $0.25^{\circ} \mathrm{C} / \mathrm{min}$ rate. The resulting array of heating and cooling spectra was combined, a corresponding array of empty-crystal spectra was subtracted from it, an ATR correction was performed, and the maxima positions were determined. The sample was in the ambient atmosphere during the measurement. The standard method is finding the x-position of the interpolated maximum or minimum. The intensity is the corresponding $y$-value of the interpolated maximum or minimum. The sensitivity parameter was 5-20\%. The higher the threshold is, the fewer bands are displayed.

\section{Results and Discussion}

\subsection{Band assignment}

Most bands belong to quartz lattice (797 and $775 \mathrm{~cm}^{-1}$ [shoulder], O-Si-O stretch, 697, 535, $510 \mathrm{~cm}^{-1}$, Si-O-Si bend; 460, 450 (shoulder), 430, 420, and $410 \mathrm{~cm}^{-1}$, O-Si-O bend. Bands at 750 and $455 \mathrm{~cm}^{-1}$ are amorphous silica species. Broad bands with maxima at 640 and $610 \mathrm{~cm}^{-1}$ are water librations. A broad weak band at $1260 \mathrm{~cm}^{-1}$ is quartz combination band $\left(\cong 460+795 \mathrm{~cm}^{-1}\right)$; a broad band at $1120-1070 \mathrm{~cm}^{-1}$ is O-Si-O stretch in crystalline and amorphous $\mathrm{SiO}_{2}$ species; bands at 1035 and 1010, quartz lattice $\mathrm{O}-\mathrm{Si}-\mathrm{O}$ stretch; $1115,93 \mathrm{~cm}^{-1}$ 7, amorphous $\mathrm{SiO}_{2} \mathrm{O}-\mathrm{Si}-\mathrm{O}$ stretch (not present in quartz); 912 , amorphous silica [8]; 890, 875. Weak bands at 855, 845 belong to Al-OH [9]. The band at $1645-1640 \mathrm{~cm}^{-1}$, shoulder band, bend $\left(\mathrm{v}_{2}\right)$ of the covalent bonds of liquid absorbed water [10]. The complex, broad band at $1580 \mathrm{~cm}^{-1}$ is an antisymmetric carboxylate stretch, with contributions from hydrogen-bonded $\mathrm{SiOH} \ldots \mathrm{H}_{2} \mathrm{O} \mathrm{HO}-\mathrm{H}$ stretch (amorphous) [11]. A broad band at $1395 \mathrm{~cm}^{-1}$ is the symmetric carboxylate stretch. The range $3100-2800 \mathrm{~cm}^{-1}$ is comprised of bands at 3020 (3020-3100), alkene/aromatic sp ${ }^{2}=\mathrm{CH}_{2}$ stretch; 2970 and $2880 \mathrm{~cm}^{-1}$, the antisymmetric and symmetric stretch of methyl groups; 2925 and $2850 \mathrm{~cm}^{-1}$, the antisymmetric and symmetric stretch of methylene groups, bands of $\mathrm{CH}_{\mathrm{x}}$ groups are on the shoulder of the $\mathrm{OH}$ continuum band. Other bands are 3710 (unbonded $\mathrm{SiO}-\mathrm{H}$ stretch, tilted (kaolinite, clay) [11]; 3700-3680, hydrogenbonded $\mathrm{SiO}-\mathrm{H} \ldots \mathrm{H}_{2} \mathrm{O}$ stretch (amorphous species); 3665, 3655, 3645, 3635, 3610 non-silicate $\mathrm{OH}$; 3650, 3630 isolated SiO$\mathrm{H}$ in quartz; and 3400 and 3290 condensed-phase antisymmetric and symmetric hydrogen-bond ensembles. 


\subsection{Temperature dependence}

The overall course of changes in IR spectra of soils is illustrated by Figure 1 (left). Most bands in the range 1250-400 $400 \mathrm{~cm}^{-1}$ are lattice vibrations of quartz and other structured inorganic matrix components. The lattice bend vibrations of Siof Si-O-Si $\left(510 \mathrm{~cm}^{-1}\right)$ and $\mathrm{O}-\mathrm{Si}-\mathrm{O}\left(460,450\right.$, and $\left.430 \mathrm{~cm}^{-1}\right)$ do not show any shifts following the behavior of first-order order fundamental vibrations. On the contrary, lattice $\mathrm{O}-\mathrm{Si}-\mathrm{O}$ stretch vibrations at 797 and $775 \mathrm{~cm}^{-1}$ exhibit a redshift of of $0.4 \%$, which is smaller than bands, the band at 775 is present as a shoulder. The band at $535 \mathrm{~cm}^{-1}$ experiences a redshift, redshift, indicating a 2nd order (overtone) band of the primary lattice vibration at $263 \mathrm{~cm}^{-1}$. The same can be assumed for for the intense band at $697 \mathrm{~cm}^{-1}$ (Figure 1) as it has a counterpart lattice vibration at 398-400 $\mathrm{cm}^{-1}$ [12]. The band at $750 \mathrm{~cm}^{-}$ ${ }^{1}$ does not shift and does not belong to quartz lattice frequencies but amorphous silica species. All the bands attributed to quartz show the temperature behavior not different from quartz samples (bands at 365,697 , and $775 \mathrm{~cm}^{-1}$ ). However, in the case of the $\mathrm{SiO}_{2}$ lattice band at $1163 \mathrm{~cm}^{-1}$ [12], the temperature dependence corresponds to quartz lattice changes [13] was found for the large fractions. However, for the fractions of $50 \mu \mathrm{m}$ and below, the temperature behavior of the band starts to deviate from the quartz showing a larger redshift (Fig. 1), which can be the manifestation of the effect of SOM or more cracked structures of the mineral matrix in these structures $[13,14]$.
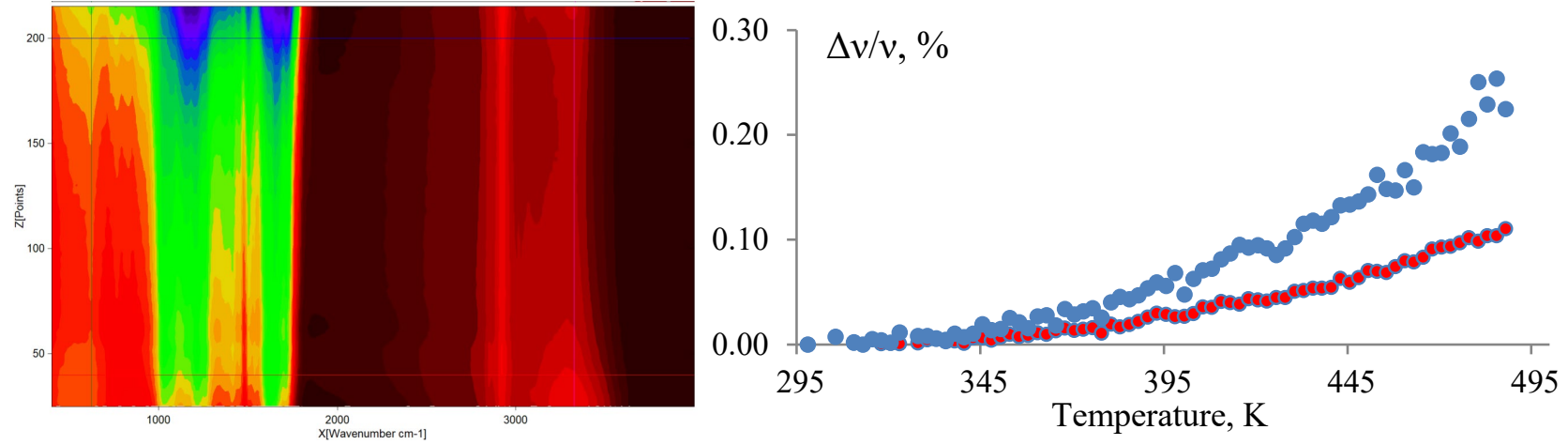

Fig. 1: The temperature spectra of soddy podzol soil (left) vertical axis is temperature, $\mathrm{C}$; and relative changes in the band position at $1163 \mathrm{~cm}^{-1}$ for quartz (red) and native steppe chernozem (blue) fractions of $50 \mu \mathrm{m}$ (right).

The band at $715 \mathrm{~cm}^{-1}$ cannot be attributed to any lattice bands of quartz or silicate minerals, and its position does not change with temperature so that it can be the manifestation of SOM, in-phase rock $\mathrm{CH}_{2}$ vibrations of $\mathrm{C}_{4}$ alkanes [15].

Condensed-phase antisymmetric $\left(3400 \mathrm{~cm}^{-1}\right)$ and symmetric $\left(3290 \mathrm{~cm}^{-1}\right)$ hydrogen-bond ensembles behave as predicted from the theory of fundamental vibrations: the energy of antisymmetric vibrations increases and the energy of symmetric vibrations decreases [16] monotonously. The band at 3695 shows a complex behavior with a general trend in the energy increase. This band experiences the most considerable shift by modulus (by $10 \mathrm{~cm}^{-1}$ ) among all the spectrum bands. Also, this band shows the strongest redshift. The shift in the band at 3695 is accompanied by the appearance of weak bands at 3710 and 3715 that can be attributed to unbonded $\mathrm{SiO}-\mathrm{H}$ stretch, tilted vibrations in amorphous silica [11]. A band at 3620 experiences a mirror-like behavior synchronous with the band at $3695 \mathrm{~cm}^{-1}$. Both bands at $3700-3680$ and 3620 are observed in kaolinite and attributed to amorphous and quartz $\mathrm{SiO}-\mathrm{H}$ bands.

\section{Conclusion}

Thus, when heated in the range $25-215^{\circ} \mathrm{C}$ in air, the IR spectra of studied soils undergo several changes. Almost all bands, both belonging to mineral fragments and purely organic, shifted in frequency, and this effect is reversible and, when cooled, the bands return to their original frequency. Both the band shift scale and the various band dependence on temperature differ significantly. Some bands demonstrate almost wholly the same temperature-induced changes when comparing the samples, while other bands behave differently. The different behavior of the lattice vibrations of quartz, hydrogen bonds, and organic matter was found and discussed to differentiate soils. The proposed approach has a drawback associated with 
the duration of measurements. However, it is comparable to the duration of thermogravimetric measurements and, in principle, can be further reduced by optimizing IR measurement conditions, which may be a subject of next studies.

\section{Acknowledgements}

This work was supported by the Russian Science Foundation, grant no. 19-13-00117. This research was performed according to the Development program of the Interdisciplinary Scientific and Educational School of Lomonosov Moscow State University, "The future of the planet and global environmental change".

\section{References}

[1] A. Imeson, Desertification, Land Degradation and Sustainability. Wiley, 2012.

[2] Q. d. Jong van Lier and A. Durigon, "Soil thermal diffusivity estimated from data of soil temperature and single soil component properties," Revista Brasileira de Ciência do Solo, vol. 37, pp. 106-112, 2013. [Online]. Available: http://www.scielo.br/scielo.php?script=sci arttext\&pid=S0100-06832013000100011\&nrm=iso.

[3] G. Józefaciuk, C. Sławiński, R. T. Walczak, and A. Bieganowski, Review of current problems in agrophysics. Inst. of Agrophysics PAS, 2005.

[4] P. Falkowski, R. J. Scholes, E. Boyle, J. Canadell, D. Canfield, J. Elser, N. Gruber, K. Hibbard, P. Högberg, S. Linder, F. T. Mackenzie, B. Moore III, T. Pedersen, Y. Rosenthal, S. Seitzinger, V. Smetacek, and W. Steffen, "The Global Carbon Cycle: A Test of Our Knowledge of Earth as a System," Science, vol. 290, no. 5490, pp. 291-296, 2000, doi: 10.1126/science.290.5490.291.

[5] J. E. Katon and D. B. Phillips, "Infrared Spectroscopy at Subambient Temperatures," Appl. Spectrosc. Rev., vol. 7, no. 1, pp. 1-45, 1973/01/01 1973, doi: 10.1080/05704927308060368.

[6] D. S. Volkov, O. B. Rogova, M. A. Proskurnin, Y. R. Farkhodov, and L. B. Markeeva, "Thermal stability of organic matter of typical chernozems under different land uses," Soil and Tillage Research, vol. 197, p. 104500, 2020/03/01/ 2020, doi: 10.1016/j.still.2019.104500.

[7] I. V. Kuznetsova, "Changes in the physical status of the typical and leached chernozems of Kursk oblast within 40 years," Eurasian Soil Science, vol. 46, no. 4, pp. 393-400, 2013, doi: 10.1134/s106422931304008x.

[8] J. Bock and G.-J. Su, "Interpretation of the Infrared Spectra of Fused Silica," Journal of the American Ceramic Society, vol. 53, no. 2, pp. 69-73, 1970, doi: 10.1111/j.1151-2916.1970.tb12012.x.

[9] A. Inoue and T. Watanabe, "Infrared Spectra of Interstratified Illite/Smectite from Hydrothermally Altered Tuffs (Shinzan, Japan) and Diagenetic Bentonites (Kinnekulle, Sweden)," Clay Science, vol. 7, no. 5, pp. 263-275, 1989, doi: 10.11362/jcssjclayscience1960.7.263.

[10] J. J. Max and C. Chapados, "Isotope effects in liquid water by infrared spectroscopy. III. H2O and D2O spectra from 6000 to $0 \mathrm{~cm}(-1)$," J. Chem. Phys., vol. 131, no. 18, p. 184505, Nov 14 2009, doi: 10.1063/1.3258646.

[11] P. J. Heaney, A. K. Kronenberg, C. T. Prewitt, and G. V. Gibbs, "Chapter 4. Hydrogen Speciation and Chemical Weakening of Quartz," in Silica: De Gruyter, 1994, pp. 123-176.

[12] W. G. Spitzer and D. A. Kleinman, "Infrared Lattice Bands of Quartz," Physical Review, vol. 121, no. 5, pp. 1324-1335, 03/01/ 1961, doi: 10.1103/PhysRev.121.1324.

[13] K. L. Day, "Temperature Dependence of Mid-Infrared Silicate Absorption," The Astrophysical Journal, vol. 203, p. L99, January 01, 1976 1976. [Online]. Available: https://ui.adsabs.harvard.edu/abs/1976ApJ...203L..99D.

[14] A. M. Hofmeister and J. E. Bowey, "Quantitative Infrared Spectra of Hydrosilicates and Related Minerals," Mon. Not. R. Astron. Soc., vol. 367, no. 2, pp. 577-591, 2006, doi: 10.1111/j.1365-2966.2006.09894.x.

[15] J. Workman, The Handbook of Organic Compounds, Three-Volume Set: NIR, IR, R, and UV-Vis Spectra Featuring Polymers and Surfactants. Elsevier Science, 2000.

[16] Y. Mikhaylova, G. Adam, L. Häussler, K. J. Eichhorn, and B. Voit, "Temperature-dependent FTIR spectroscopic and thermoanalytic studies of hydrogen bonding of hydroxyl (phenolic group) terminated hyperbranched aromatic polyesters," Journal of Molecular Structure, vol. 788, no. 1, pp. 80-88, 2006/05/08/ 2006, doi: https://doi.org/10.1016/j.molstruc.2005.11.020. 